\title{
Solving the Measurement Problem and then Steppin' Out over the Line Riding the Rarest Italian: Crossing the Streams to Retrieve Stable Bioactivity in Majorana Bound States of Dialy zed Human Platelet Lysates
}

\author{
Mark Roedersheimer*
}

Research, GITE/Burn Surgery, UC Denver, USA

\begin{abstract}
Exhaustive dialysis (ED) of lysed human platelets against dilute $\mathrm{HCl}$ yields stable angiogenic activity. Dialysis against a constrained external volume, with subsequent relaxation of the separation upon opening the dialysis bag, produces material able to maintain phenotypes and viability of human cells in culture better than ED material. Significant graded changes in MTT viability measurement tracked with external volume. The presence of elements smaller than the MW cutoff, capable of setting up cycling currents initiated by oriented flow of $\mathrm{HCl}$ across the membrane, suggests that maturation of bioactivity occurred through establishment of a novel type of geometric phase. These information-rich bound states fit recent descriptions of topological order and Majorana fermions, suggesting relevance in testing Penrose and Hameroff's theory of Orchestrated Objective Reduction, under conditions more general, and on finer scales, than those dependent on tubulin protein. The Berry curvature appears to be a good tool for building a general field theory of physiologic stress dependent on the quantum Hall effect. A new form of geometric phase, and an associated "geometric" quantum Hall effect underlying memory retrieval, dependent on the rate of path traversal and reduction from more than two initial field influences is described.
\end{abstract}

Keywords: Acidity, Alzheimer's disease, caveolin-1, cystatin c, ebola, geometric phase, hemodialysis, majorana fermion, metallic hydrogen, platelet lysate, quantum hall effect.

\section{INTRODUCTION}

To every differentiable symmetry generated by local actions, there corresponds a conserved current. -concise statement of Noether's Theorem, from the wiki page

Humans can receive information contained in light and sound waves, by pathways that do not require the eyes or ears. The tendency to simplify information received, exemplified in the evolution of eyes and ears, to support efficient working models, underlies basic cognitive functions allowing a focus of attention on the most critical stimuli. Assuming that these models must be the same across members of a species misses the larger point that survival requires caution in handling information. If a chess player has been using a certain set of moves advantageously, that an opponent learns to read, and then counter effectively, knowledge of the maneuvers prior to this point of transition in the game have little relevance to future dynamics. A clear example that makes this point is the emergence of antibiotic

*Address correspondence to this author at the Research, GITE/Burn Surgery, UC Denver, USA; E-mail: emergentbiologics@gmail.com resistance. The question is, how does an organism determine the most critical stimuli to focus on, when the ecosystem possesses an effectively unbounded number of dynamic influences, and only a few must be grasped and acted on rapidly, such as the molecular signatures of a deadly pathogen about to establish an infection?

The thesis of this paper is that orientation of attention, and consequent retrieval of memory necessary to address critical field influences, effectively stresses, responsible for the orientation, can be seen to occur through establishment of a purely geometric phase, dependent initially on more than two field inputs, and also the rate of path traversal, distinguishing it from the Pancharatnam-Berry phase [1]. While individual macromolecules, such as DNA, or proteins, such as tubulin, or small factors like ATP or H+, clearly play a role in these processes, the theory views these as only discrete aspects of deeper, underlying wave patterns fitting the time-independent Schrödinger equation for bound states. A pure "wave" view allows resolution of problems associated with breakdown of "causality," typical of particle representations, if the associated paradoxes are just seen to reflect inadequate access to information, a natural aspect of the filtering done by living systems because of limited resources. This is a way of asserting the validity of "hidden variable" views to quantum mechanics, in line with those of 
Einstein, Schrödinger, and de Broglie-Bohm. In this paper the wave function is interpreted as a description of real, physical (not probabilistic) processes that contain memory in living systems. The only "indeterminacy" that derives from this view is in development of perceptual prowess, through valid assays, to access desired information.

\subsection{A Platelet Lysate Model of Stress-induced Bioactivity Control}

Platelets are a-nucleate cells of the blood essential to wound healing that contain diverse molecules expressed in the course of stress responses [2]. Clinicians typically worry if counts get low, because of the risk of internal hemorrhage. Under conditions of adequate platelet count, coagulation at sites of tearing in the endothelial layer resulting from normal stresses, where platelets would localize in clot plugs, presumably provide sufficient activity to restore vessel wall integrity. Considered as a dynamically porous sieve, the clot can allow some elements released to remain close to the wound surface, and others to diffuse out into the blood stream. Thus, critical aspects of the net bioactivity created near the vascular wall may result from transport properties tuned by interactions with the clot matrix, defined to a first approximation, by molecular weight or charge, in direct analogy with principles of gel chromatography. The dynamic aspect of these interactions suggest mechanisms for storage and retrieval of memory, present non-locally in the tissues of the organism, amounting to latent images of responses that exist to be retrieved in the presence of stressors.

If sufficient symmetry can be established using field interactions evolved against, and into the organism, that effect retrieval of memory needed to deal with stressors, such as angiogenic or antimicrobial activity from platelets, or collagen polymer formation, and relevant changes in these activities are measurable in endpoint properties of isolates made by application of the same field influences, then control and honing of the activity should be possible using Monte Carlo modeling techniques, directly analogous to methods for controlling neutron scattering interactions in nuclear reactors. Success requires a valid endpoint metric for the activity, and could be a measure of Ebola virulence suppression, or any pathogen, in culture. "Valid" means the measure tracks to higher-level responses, such as those observed in humans infected with the pathogen, AND the property sought actually exists in the mixture of factors that are being assayed.

Generally, systems of evolved biological complexity cannot be strictly understood simply in terms of variables such as energy, time, position, momentum, etc., because they yield little insight into the critical emergent properties that account for survival.

The state of an organism in the course of successfully defeating a viral pathogen could yield clues to field influences that are required to shape the necessary effect, such as a temperature profile. This would start as a retrieval of general "virus fighting" memory evolved into the organism, in the case of viruses one has never encountered. The final annealing of the factors into a state highly specific to the threat would occur in vivo when directly influenced by the potentially unlimited number of field effects resulting from the pathogen's assault patterns. This methodology could also reveal properties of resistance to a virus after surviving exposure, leading to better methods of processing the platelet extracts of those unexposed into improved states for treating the virus. This can be seen as implying a kind of 2 , or "public"-key encryption mechanism in responses to stress, since relevant influences clearly extend beyond those solely inside the organism, including gravity, light, gases, foods and liquids, or the opportunity to get good sleep covered by a warm blanket.

We have previously reported that platelet lysates dialyzed to exhaustion against $10 \mathrm{mM} \mathrm{HCl}$ maintain stability at $4^{\circ} \mathrm{C}$ and display an enhancement of dose-dependent angiogenic activity in vivo relative to undialyzed fractions [3, 4]. Removal of low MW elements, and diffusion of dilute acid into the space retaining higher molecular weight factors suggest the relevance of the model to context specific development of bioactivity at sites of wounding, or tumor stroma. Another dialysis study of ours documented effects on the final form of collagen gel assembly occurring under the conjoined, oriented influences of gravity and buffer ion diffusion into the acidified soluble phase across the membrane [5]. The finding of a distinct transition in morphology $3.4 \mathrm{~mm}$ away from the membrane in 1-g might be seen as evidence of a Pancharatnam-Berry phase, as it was lacking in gels assembled in microgravity. Because collagen clearly evolved as a response to gravity, this system suggests a way to build highly sensitive interferometers for gravity waves, as it can be expected that the point of transition will be sensitive to changes in collagen concentration, temperature, MW cutoff of the membrane, MW of buffer ions, and container geometry. Overall, these findings suggest that simple separations can regulate emergence of critical activities nascent in tissues such as platelets, or those rich in collagen, including, but certainly not limited to, bone, cartilage, and tendon, revealing the role of self-similarity in evolved stress responses.

Platelets contain bone morphogenetic proteins (BMPs) [6], members of the TGFbeta superfamily that track to the origins of multicellular life forms [7]. Variation in BMP levels between donors, as well as $\mathrm{pH}$ dependence of release has been reported [8]. Activation in vivo by denaturing conditions including extremes of $\mathrm{pH}$ [9], suggests their relevance to stress response. The designation "body morphogenetic proteins" has been proposed because of their involvement with so many developmental processes [10]. A dramatic example of the conserved nature of these factors is the demonstration that decapentaplegic, a Drosophila homoloque of mammalian BMP-2/-4, will induce endochondral bone formation in a mammal [11]. Considering that cells in vivo and in culture are also widely known to be highly sensitive to $\mathrm{pH}$, the better focus for control of these well-documented activities should now be acidification, rather than the proteins themselves, as evolution will have recognized the expediency, and energy savings, of adjusting flows of protons, rather than the expression of large protein molecules, at times of serious stress. 


\subsection{The Case for Superconductivity, Metallic Hydrogen, and Majorana Fermions in Living Systems}

It is noteworthy that transition temperatures of the copper oxides, the first "high temperature" superconductors discovered, and the later iron-based composites, straddle the boiling points of liquid oxygen and nitrogen, far below the sublimation point of carbon dioxide [12]. A shift in transport of total available electrons through iron, relative to copper, at lower temperatures, along with supportive phase transitions of $\mathrm{O} 2$ and $\mathrm{N} 2$ in a complex aqueous media cyclically exposed to sunlight, suggests a means of thermoregulation by the environment of early life forms, through direct activation of iron-dependent energy production mechanisms. The fact that copper-dependent amine oxidases (CDAOs) eliminate diverse biogenic amines and are induced in states of neuronal injury [13], suggest the foundational role of copper-based electron transport in regulation of drive, needed at higher temperature, to address the imminent "eat, or be eaten" reality of each new dawn. The known hygroscopic character of copper oxides suggests the limited relevance of methods to measure the superconductivity of such materials, if they cannot do so in the presence of water. [relevance noted on superconductors.org website, accessed online March 26, 2015.] .

Living tissues can easily relax restrictions on spin, charge and geometry, that hinder creation of theoretical superconducting states in solid metals [14], such as conjoined forms of two electrons ( $\mathrm{p}$-waves), since they possess freely mobile proton and divalent cation phases, most notably $\mathrm{Ca}++$, that could stabilize these "exotic" -2 , spin 1 electron states. Coalescence of protons and electrons into similarly "exotic" forms is also conceivable given their proximity in overlapping mobile phases, and presence of many negatively charged groups on macromolecules supportive of low velocity, and therefore long de Broglie wavelength proton states, in vivo. The value of these types of states could have driven evolution of controls in living systems for regulation of energy metabolism through diffuse ordering effects, manifesting as "superatoms," in leaves responding to sunlight, through "optical molasses"-type influences, such as used to create Bose-Einstein condensates [15].

The initial product of water-splitting reactions from sunlight, with highest value to early life forms, in a setting of low oxygen, could have been metallic hydrogen, based on a BCS theory model of a stable phase at $95^{\circ} \mathrm{C}$ possessing properties of superconductivity [16]. Given an extraordinary energy density, it would be the ideal energy currency in hot springs near thermal vents, eventually driven out by the accumulation of oxygen. Beneficial effects of molecular hydrogen documented in a large number of diseases, with no clear dose-dependence [17], in line with a hormetic response, could suggest a secondary role in sensing, and clearance of the metallic form, if low oxygen states characteristic of metabolic dysfunction support accumulation of toxic levels in disease states.

Limitation of metallic phase formation by access to nucleons from water hydrogen atoms could underlie toxicity of heavy water in Eukaryotes [18]. Accordingly, increased toxicity in malignant cells [18] and near absence of toxicity in prokaryotes [18], may suggest the role of metallic hydrogen in diverse, chronic inflammatory states of oxygen dependent organisms susceptible to infections by prokaryotes. Removal of excess metallic hydrogen in a deep cleansing breath, reflected in levels of molecular hydrogen in the alveoli, would be difficult to confirm with current analytical methods. Generation of metallic hydrogen could control state shifts in plants, if diffusion through the outer membrane supports immediate conversion into the molecular form.

A obviating study documenting lack of significant physiological response, most prominently mtDNA/nDNA, in subjects aged 37-64 participating in a 6 week study of exercise and protein intake who consumed D20 for labeling purposes, showed a strikingly consistent VO2max bump of approximately $10 \%$ [19] suggesting a direct chemicophysical response in this setting. This can be explained if metallic phase formation is constrained by access to nucleons (protons and neutrons) from water that is relieved by consuming $\mathrm{D} 2 \mathrm{O}$, potentially obviating any need for a physiologic adaptation to the exercise regimen. It would be interesting to know if percent deuteration achieved in each subject, reported only as the group average, $1.5-2.5 \%$ [19, Fig. 3], tracked with VO2max, as a tight correlation would be expected if enhanced metallic phase formation underlies the effect. If phase formation involves beta decay, this may account for the natural role of Aluminum, as it is a good absorber of beta particles. Accordingly, aluminum may regulate transition of nucleons into the metallic phase, explaining toxicity at high levels.

The first Quantum corrals were made of iron adatoms on a copper surface allowing the mirage effect to be generated by placing cobalt in the corral [20].

It is noteworthy that cytochrome c oxidase possesses two iron and two copper centers [21]. Amyloid precursor protein has been shown to possess amine oxidase activity inhibited by zinc [22]. Copper can support self-assembly of Myelin basic protein [23] and large conformational changes in Alpha-synuclein upon binding [24]. The gentle production of $\mathrm{H} 2 \mathrm{O} 2$ from PrP binding copper in a high occupancy mode has been interpreted to suggest involvement in cellular signaling mechanisms [25]. A theory suggesting the mirage effect only requires "an arrangement of adatoms or other defects that lead to a buildup of surface state electron amplitude at two locations within the coherence length of the electron" [26, p. 14] gives a clue to the role of $\mathrm{Fe}$ and $\mathrm{Cu}$ binding in these metalloproteins that are capable of assuming variable conformations. Accordingly, [O2] oscillation can determine configurations supporting a mirage effect that controls gating of electron flow toward oxidation of potent amines, or iron-based energy metabolism, as needed outside neurons.

$\mathrm{CoCl} 2$ can turn on erythropoetin (EPO) production under normoxic conditions [27]. In cells depleted of mitochondrial DNA in which hypoxia will not generate ROS, or induce expression of EPO, application of $\mathrm{CoCl}_{2}$ will increase ROS generation and maintain viability to induce expression of EPO [28]. Methionine aminopeptidase 2, a target of antiangiogenic therapies, is on a short list of known cobaltoproteins [29]. Based on these reports, and the 
viability of Cobalt to disperse electron probability amplitude constrained in a quantum corral formed between copper and iron [20], it appears to be capable of controlling electron transport gating mechanisms established before oxygen was abundant on the planet, but still essential during states of low oxygen, typical of those driving angiogenic responses. The creation of a $\mathrm{NaCoO}_{2}$ compound with superconducting properties dependent on the presence of water [30] is consistent with a capacity of cobalt to regulate superconducting states under physiologic conditions.

An investigation of the utility of metal-organic frameworks (MOFs) for deuterium isotope separation established the superiority of a $\mathrm{Zn} / \mathrm{Cl} \mathrm{MOF}$ in terms of high selectivity, and support of quantum sieving up to $60 \mathrm{~K}$, whereas a $\mathrm{Co} / \mathrm{Cl} \mathrm{MOF}$ was remarkable in supporting a small pore structure, low selectivity, but high binding capacity at $30 \mathrm{~K}$ [31]. The author suggests that the Co based material could be used advantageously in an initial step, followed by a second step with a material of higher selectivity [31, p. 134]. Maintenance of higher selectivity of the $\mathrm{Zn} / \mathrm{Cl}$ MOF up to $50 \mathrm{~K}$, compared to $\mathrm{Co} / \mathrm{Cl} \mathrm{MOF}$ at $30 \mathrm{~K}$ [31, Fig (5). 14, p. 133], suggests a basis for a gating mechanism between metallic and molecular hydrogen phases dependent on differential deuterium concentrating effects of $\mathrm{Co}$ and $\mathrm{Zn}$ in a pre-oxygenic ecosystem. This is directly parallel to that proposed earlier for $\mathrm{Fe}$ and $\mathrm{Cu}$ in an oxygen-dependent ecosystem, through exploitation of temperature-dependent electrical superconductivity controlled by cyclical exposure to sunlight.

Living organisms should be seen as the ideal setting in which states of "non-trivial emergent excitation," characteristic of a Majorana Fermion [14], may be found along with evidence of metallic hydrogen. This would amount to a complex waveform with the highest frequency components controlled by interconversion of water nucleons and electrons between states of molecular and metallic hydrogen, potentially accounting for enormous information, and energy storage capacity. The mode has been described as having the potential to support "fault-tolerant computation" [14]. This author notes that while deducing existence of a Majorana mode tells you nothing about the encoded qubits, retrieval of the information should occur if two of them are brought into contact [14, p. 26].

This report presents results of an experiment in which this appears to have occurred in the course of dialyzing platelet lysates against dilute acid, within a constrained volume, followed by fusion of the separated phases when one end of the bag was opened while still submerged in the larger volume. Analysis of these final states revealed a graded change in quantitative indices of regenerative activity in human cell culture suggesting they represent stable Majorana bound modes.

\section{METHODS}

Three units of expired platelets (A, B, C) were obtained by courier mid-afternoon of the day following midnight expiration. These were aliquoted within a laminar flow hood into $50 \mathrm{ml}$ conicals and pelleted using a benchtop centrifuge at low speeds $(2000,2500,2500 \mathrm{rpm})$ for 20,25 , and 20 minutes, respectively, to pellet the platelets. The serum was pipetted off each pellet with care to avoid disruption. A total of approx $90 \mathrm{mls}$ of sterile water was distributed equally on top of the pellets, for each unit. The batches were placed in a benchtop bath sonicator for 60,60 , or 40 secs, respectively. Following a second centrifugation at $3000 \mathrm{rpm}$, for 25 minutes, the supernatants were collected and re-pooled. At this point material from each unit was divided and either exhaustively dialyzed (ED), by putting $40 \mathrm{ml}$ of the isolate in a 6-8kD dialysis bag, and carrying through two $1 \mathrm{~L}$ exchanges of $10 \mathrm{mM} \mathrm{HCl}$ over a 2-day period, or taken through a "constrained" dialysis (CD) process. Each involved overnight refrigeration with gentle agitation or stirring. For CD the $40 \mathrm{ml}$ isolate was placed into a 6-8 $\mathrm{kD}$ dialysis membrane against either 300,350 , or $400 \mathrm{ml}$ of 10 $\mathrm{mM} \mathrm{HCl}$. The following day the material inside the bag, and in the larger dialysis solution, were allowed to gradually reintegrate by opening one of the clips and placing back in the container, with removal of the bag an hour, to a few hours later. Test strips were used to assess $\mathrm{pH}$ for each isolate. Materials were sterile filtered through standard low protein binding membranes, and maintained stability (i.e. showed no overt signs of precipitation) at $4 \mathrm{C}$ during the test period. Base media was DMEM with $4.5 \mathrm{gm} / \mathrm{L}$ glu,+lglutamine, and $110 \mathrm{mg} / \mathrm{ml}$ sodium pyruvate.

Normal, human dermal fibroblasts were plated at $60,000 /$ well in a 96 well plate in a volume of 300 microliters. After one day, base media was changed to include the test media. Feeding regimen was Mon, Wed, and Fri. Standard mammalian cell culture incubation conditions were used. An MTT assay (Cayman Chemical Co, Ann Arbor, MI item 10009365) was run at 7 days. Visual assessment indicated approximately identical cell number as on the day of plating.

Ranked evaluation of the following was done from the images: 1) the relative number of "-cyte" to "-blast " forms on a total 4-cross scale (distributed across "f-cytes" or "fblasts" columns); 2) the degree of differentiation of the blast forms overall, in terms of degrees of branching, distinct nuclei and prominent rough ER, on a 2-cross scale, 3) thickness of diffuse matrix, on a 3-cross scale, 4) amount of aligned fibrils on a 3 -cross scale.

Since CD involves dilution by solution outside the bag, ED material was diluted comparably (10x) using vehicle (10 $\mathrm{mM} \mathrm{HCl}$ ), prior to analysis. An accounting of mass loss was done from a report of $2.6+/-0.6 \mathrm{pg} / \mathrm{plt}[32]$ and $3 \times 10^{11} \mathrm{plts}$ in a typical unit yields a total protein content of $780 \mathrm{mg} / \mathrm{unit}$. A typical finding of 4-7 (ave. 5.5) $\mathrm{mg} / \mathrm{ml}$ by Bradford assay from a single unit in 70-90 mls (ave. 80) yields $(5.5 \mathrm{x} 80 \mathrm{mls}$ =) $440 \mathrm{mg} /$ unit. Pellet size after the second, higher speed spin is typically more than half original pellet prior to extraction, so a large fraction (on order of $>85-90 \%$ ) of the total soluble protein appears to be accounted for in the ED sample. Percent additions were reported, as protein mass has been a weak predictor of ED angiogenic activity in the past.

One-way ANOVA was done on ED samples to evaluate resulting inter-unit variation, and on $\mathrm{CD}$ samples to evaluate 
Table 1. MTT Assay - Group designations of platelet extract supplemented media.

"Un-numbered" control group - 20\% animal serum supplemented DMEM
1) Exhaustive process, $1 \mathrm{x}$, donor unit $\mathrm{A}, 2 \%$ in DMEM
2) Exhaustive process, $1 \mathrm{x}$, donor unit $\mathrm{B}, 2 \%$ in DMEM
3) Exhaustive process, $1 \mathrm{x}$, donor unit $\mathrm{C}, 2 \%$ in DMEM
4) Exhaustive process, $x / 10$, donor unit $A, 4 \%$ in DMEM
5) Exhaustive process, $x / 10$, donor unit $B, 4 \%$ in DMEM
6) Exhaustive process, $\mathrm{x} / 10$, donor unit $\mathrm{C}, 4 \%$ in DMEM
7) Exhaustive process, $\mathrm{x} / 10$, donor unit $\mathrm{A}, 8 \%$ in DMEM
8) Exhaustive process, $\mathrm{x} / 10$, donor unit $\mathrm{B}, 8 \%$ in DMEM
9) Exhaustive process, $\mathrm{x} / 10$, donor unit $\mathrm{C}, 8 \%$ in DMEM
10) Constrained process, $1 \mathrm{x}$, donor unit $\mathrm{A}, 4 \%$ in DMEM (ext. vol $=300 \mathrm{ml}$ )
11) Constrained process, $1 \mathrm{x}$, donor unit $\mathrm{B}, 4 \%$ in DMEM (ext. vol $=350 \mathrm{ml}$ )
12) Constrained process, $1 \mathrm{x}$, donor unit $\mathrm{C}, 4 \%$ in DMEM (ext. vol $=400 \mathrm{ml}$ )
13) Constrained process, $1 \mathrm{x}$, donor unit A, $8 \%$ in DMEM (ext. vol $=300 \mathrm{ml}$ )
14) Constrained process, $1 \mathrm{x}$, donor unit $\mathrm{B}, 8 \%$ in DMEM (ext. vol $=350 \mathrm{ml}$ )
15) Constrained process, $1 \mathrm{x}$, donor unit $\mathrm{C}, 8 \%$ in DMEM (ext. vol $=400 \mathrm{ml}$ )

Table 2. MTT assay values, normalized off blanks.

\begin{tabular}{|c|c|c|c|}
\hline 20\% DMEM & 0.85 & 1.24 & 0.91 \\
\hline 1 & 0.52 & 0.55 & 0.94 \\
\hline 2 & 0.40 & 0.54 & 1.10 \\
\hline 3 & 0.42 & 0.81 & 1.14 \\
\hline 4 & 0.06 & 0.14 & 0.52 \\
\hline 5 & 0.14 & 0.10 & 0.37 \\
\hline 6 & 0.11 & 0.14 & 0.67 \\
\hline 7 & 0.05 & 0.16 & 0.99 \\
\hline 8 & 0.52 & 1.02 & 1.09 \\
\hline 9 & 0.59 & 1.13 & 1.20 \\
\hline 10 & 0.52 & 1.35 & 1.35 \\
\hline 11 & 0.16 & 1.20 & 1.30 \\
\hline 12 & 0.35 & 0.24 & 0.60 \\
\hline 13 & 0.00 & 0.89 & 0.59 \\
\hline 14 & 0.29 & 1.35 & 0.56 \\
\hline 15 & 0.09 & 0.31 & 0.24 \\
\hline
\end{tabular}

the effect of external volume changes. T-tests were done to evaluate differences between serum, ED and CD groups.

\section{RESULTS}

Materials resulting from either process had a $\mathrm{pH}$ in the range of 2-3, typical of prior results with ED. Media containing platelet lysates maintained color and clarity after addition of all extract variants, up to the point of media changes, indicating minimal effect on $\mathrm{pH}$ across all the processes used. Slight color changes were noted in the serum groups at feeding, suggesting some change in $\mathrm{pH}$ in these treatments. Since metabolic activity in fibroblasts may be directed to matrix synthesis, migration, or proliferation, to variable degrees, morphology was evaluated and correlated to MTT readings. 


\subsection{Exhaustive Dialysis (ED) Yields Little Inter-unit Variation}

MTT values resulting from old process applied to three different units were evaluated using the Null hypothesis $\mathrm{H}_{0}=$ no effective difference in resulting bioactivity across units.

$1,2,3: \mathrm{F}(2,6)=0.1249,<5.143, \mathrm{H}_{\mathrm{o}}$ accepted

4, 5, 6: $\mathrm{F}(2,6)=0.136,<5.143, \mathrm{H}_{\mathrm{o}}$ accepted

$7,8,9: \mathrm{F}(2,6)=1.795,<5.143, \mathrm{H}_{\mathrm{o}}$ accepted

Thus, ED creates consistent bioactivity across units, based on the MTT assay.

\subsection{Constrained Dialysis (CD) Volume Controls Changes in Bioactivity}

MTT values for the same three units, with graded application of the new process (dialysis against 300,350 , or $400 \mathrm{ml}$ ), pooling the $4 \%$ and $8 \%$ groups (and excluding the 0.0 in group 14 as an outlier), and evaluating the Null hypothesis $\mathrm{H}_{\mathrm{o}}=$ graded application of constrained process is not responsible for the observed variation in activity:

$10+13,11+14,12+15: \mathrm{F}(2,14)=4.06,>3.739$, so $\mathrm{H}_{\mathrm{o}}$ can be rejected.

Thus, $95 \%$ confidence can be asserted that CD creates a real effect across batches, assuming effects due to dilution are not controlling it. Upon reincorporation between the two portions this amounts to a change across the groups of roughly $26 \%(40 / 340=0.118,40 / 390=0.103$, and 40/440 $=$ 0.091 , so $(.118-0.091) /($ average dilution $=.104)=0.259)$. Separating the $4 \%$, and $8 \%$ results should reveal relevant changes, if dilution has a strong effect. A two-tailed t-test was done since no prior knowledge exists to suggest which group should be greater. Averages for the 4 and 8\% groups of 0.78556 and 0.54 , respectively, yield a p-value of 0.295 . This effect is contrary to a drop seen with the dilution across $\mathrm{CD}$ groups, suggesting that $\mathrm{CD}$ volume exerts the major effect on MTT reading.

\subsection{Gives Improved Bioactivity Compared to ED Treatment}

Applying a 2-tailed, unpaired t-test on $20 \%$ serum vs pooled $4 \%$ ED groups yields $\mathrm{p}=0.0004$. Thus, high confidence can be asserted that at 4\%, ED material performs below standard 20\% serum culture conditions. Applying a 2tailed, paired t-test on pooled $4 \%$ ED groups vs. pooled $4 \%$ $\mathrm{CD}$ groups yields a $\mathrm{p}=0.008$. Thus, high confidence can be asserted that they are different. Applying a 2-tailed, unpaired t-test on the SG vs pooled 4\% CD group yields a $\mathrm{p}=0.5$, giving no basis to claim a difference exists. These results are summarized in Fig. (1).

\subsection{Morphology evidence:}

One representative picture for each culture well was collected in a blinded fashion to document the morphology at the end of the test period. A notable finding was the appearance of a variable, fine precipitate, suggesting debris on the culture surface in the SG and groups 1-5, with conspicuous absence of this feature in groups 6-15. The possibility that this was evidence of infectious contamination was ruled out by absence of change in $\mathrm{pH}$ that would have been expected, and reflected in a change in media color and clarity.

Key features of these images included cells with either a fibrocyte (spindle cell) or a fibroblast character, the latter typified by 3 or more branched processes off a central mass with prominent nucleus and rough endoplasmic reticulum surrounding it. No marked change in cell number was noted by gross inspection during the culture period.

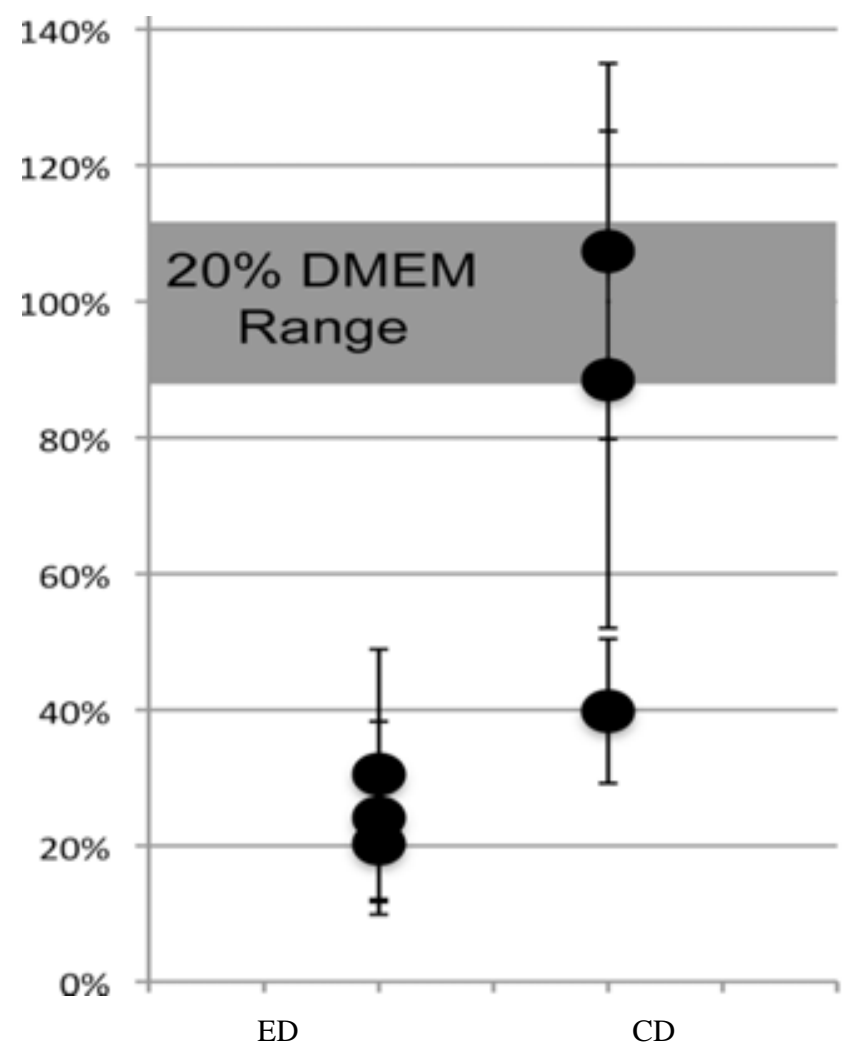

Fig. (1). MTT response comparison of $4 \%$ exhaustive dialysis (ED) to constrained dialysis (CD) process groups normalized against $20 \%$ serum.

In some groups tracks of highly aligned bands of optically homogeneous matrix were observed, with a thickness approximately equal to a fibroblast migrating over the provisional matrix surface, indicative of differentiated fibroblast behavior, in line with normal tissue remodeling [33] and approximating dermal compartment healing at 5-6 days after injury [34].

In nearly all groups a diffuse provisional matrix was noted on the culture surface as rolling variations in optical density, from which more metabolically and phenotypically robust cells can develop and form organized collagen bands in line with a synergy between growth factor and matrix influences on differentiation [33]. Type I collagen expression is a recognized marker of fibroblast phenotype [35].

The results presented in Table $\mathbf{3}$ reveal increased proportions of "-blast" to "-cyte" forms, increased diffuse matrix synthesis, and increased amounts of aligned forms, as dose of ED (groups 1-9), and CD (groups 10-15) process 
Table 3. Ranked morphological findings:

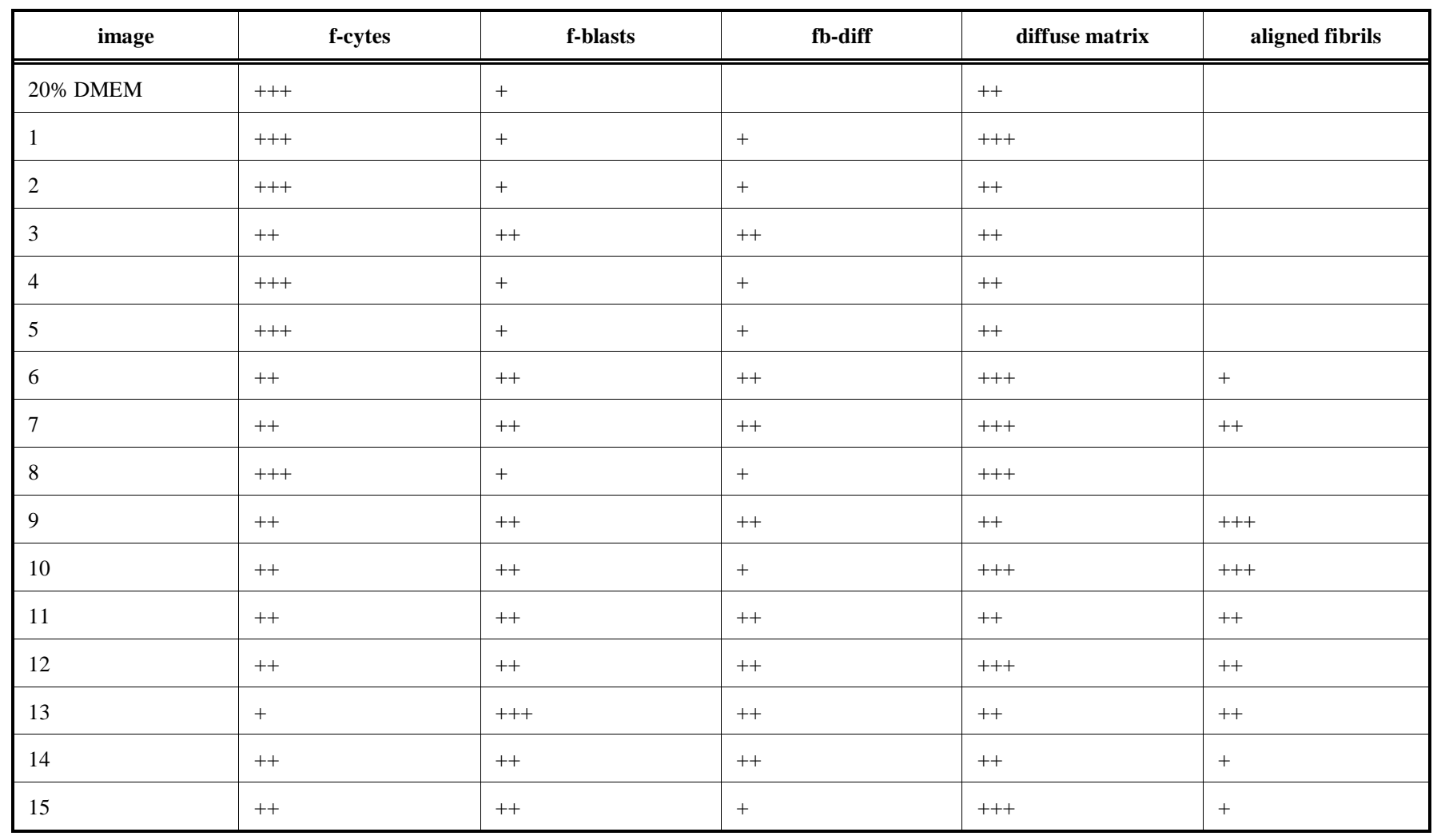

materials increase. Groups 10, 11, and 12 (4\% CD) were also superior by MTT assay (Fig. 1).

\section{DISCUSSION}

Data in this report shows how the diffusion of dilute acid across a dialysis membrane into a space containing a natural bioactive extract can change the bioactivity of the material in proportion to the external exchange volume, in a setting where major alterations in composition would not be expected. These changes were determined through visual evaluation of key phenotypic characteristics of the cells, and a quantitative MTT assay that is an appropriate measure of viability in such settings [36, p. 141]. Wound healing requires growth, and subsequent apoptosis of cells, suggesting the value of this type of effect in regulation of cellular viability, to avoid tumor formation. The effect could conceivably occur naturally across any selectively permeable membrane, such as clot structures, in vivo.

Wounding can be seen generically as disruptive or denaturing action on a tissue, resulting in breakdown of the normal relationships between soluble and insoluble phases. Lysis of a tissue, such as platelets in water, is a controllable event modeling these processes. Because survival following wounding requires retrieval of the most critical functions of the organism under conditions that disrupt cellular membranes, receptor mediated signal transduction mechanisms leading to gene expression have little relevance until the emergent processes of dispersed biomolecules, such as clotting factors, can support reestablishment of tissue organization and stable cell membranes. This is reflected in the fact that yeast lysates can still ferment [37], and collagen polymerization is controllable in the absence of cells through $\mathrm{pH}$, and gravitational influences alone [5].

Placing a lysate of platelets within a dialysis bag against a larger volume of dilute $\mathrm{HCl}$ models the initial release of factors within a clot at an ischemic wound site, setting up an asymmetric flow of charge based on the smaller weight of protons than $\mathrm{Cl}$ - ions. Release of ions from high $\mathrm{MW}$ proteins weakly denatured by the faster proton wave would lead to their redistribution across the membrane, amounting to a positive current inversion, since common protein bound ions, such as calcium, iron, zinc and copper, can assume +2 charge in vivo. Consequent disruption of CDAO activity would lead to elevated potent amine levels, that can account for initiation of responses to this well-known type of initial asymmetry, such as occurs during digestion in the stomach. Because amines typically only assume $a+1$ charge, and have higher MW than divalent cations, this asymmetry could be the basis for counterion effects that initiate looping currents able to support states of superconductivity in line with a "heavy-fermion" mode, or the FFLO state [38, 39]. These may fit descriptions of Fröhlich condensation, Orchestrated Objective Reduction, or other forms of "macroscopic quantum coherence" [40].

The initial state of charge asymmetry might also support creation of a unique variant of the quantum Hall effect (QHE), based on a derivation of the Berry curvature responding to the rate of change of an external parameter controlling physical observables that is general enough to be relevant to living systems [41]. As the Berry curvature is a 
form of susceptibility [41], a measure of the change in an extensive property (ie mass, or volume) under variation of an intensive property, such as density $(=\mathrm{M} / \mathrm{V})$ [42], the concept translates naturally into a "field" based view of stress in living systems. The description of a "dynamical" QHE appears to model a continuous variation in the external (geometric) parameter, rather than the case of three discrete values studied here. This situation is clearly more typical of circumstances in vivo. Accordingly, acquisition of Pancharatnam-Berry phase between the discrete states, would have been a continuous function of the volume change, or container geometry, at fixed volume, resulting from a "dynamical" QHE, directly analogous to stress (a curvature) applied to a living system, leading to stable states such as were defined at the discrete volumes. These effects should be observable with appropriate sampling and measurement, using an adaptation of the model that has been presented.

Our results suggest observation of a pure "geometric" QHE, as discrete, stable states resulted strictly from the geometric constraint alone. The dynamical QHE was likely involved, initiated as the dialysis bag containing the lysate entered the exchange volume, driving acquisition of a timedependent Pancharatnam-Berry phase, effectively a geometric aspect of the "stress," or Berry curvature, postulated to be relevant to the time-reversal symmetry breaking properties of such systems [41]. Models of oscillating chemical reactions, general enough to encompass biological networks, and the human nervous system, have been developed $[43,44]$. These authors emphasize examples of organization resulting from interfacial effects, photoredox cycling of iron, the role of light in driving processes far from equilibrium, and the importance of concentration, over catalysis [43].

The data presented can explain worsening of periodontal status with hemodialysis vintage [45], lack of change in levels of cystatin c between sessions [46] and superior predictive power of cystatin c for cerebral microbleeds [47], if cystatin c can act as a CDAO, or a chaperone of CDAOs. Support of amine oxidase function in the presence of copper would explain protection of neuronal cells against mutant cu/zn SOD toxicity [48] if this form of SOD also has a $\mathrm{CDAO}$ function that is disrupted by the mutation. Evidence for these functions would establish how local potent amine levels directly regulate cu/zn-SOD activity.

Cystatin c co-localizes in amyloid deposits of both nondemented aged people and those with Alzheimer's disease, and has been hypothesized to serve a protective role in $\mathrm{AD}$ [49]. Plasma semicarbazide-sensitive amine oxidase (SSAO) activity has been shown to correlate with renal dysfunction and levels of Cystatin c [50]. SSAO has been tracked to vascular adhesion protein-1 (VAP-1), a copper dependent amine oxidase, and $180 \mathrm{kD}$ glycoprotein [51]. A relationship between $\mathrm{AD}$, vascular dementia risk and vessel wall SSAO activity has been suggested [52]. A MW of only $13 \mathrm{kD}$, and co-localization at sites of deposition around neurons, may suggest cystatin $\mathrm{c}$ is expressed to address dynamic stresses, to augment function of CDAOs around neurons, and other cells. This could have been missed if copper binding and oxidase activity are simultaneously dependent on conformation, solvent environment, and copper availability. It is worth noting that copper binding to Cystatin B leads to inhibition of amyloid fibril formation [53], and Cystatin c overexpression rescues a Cystatin B mutant phenotype that causes progressive myoclonic epilepsy [54]. Tracking levels of biogenic amines such as dopamine added to physiologic solutions of variable ionic and polar character containing cystatin $\mathrm{c}$ and varying amounts of copper, might reveal, or rule out $\mathrm{CDAO}$ activity.

Leakage of fibrinogen has been found to presage microglial motility, perivascular clustering, and onset of axonal damage in a rodent model of multiple sclerosis [55]. Based on mechanisms discussed, a fibrin membrane might be acting as an insulator in these settings creating disruption of conduction mechanisms necessary for stability of axonal sheaths targeted in multiple sclerosis. Fibrin gel networks have been shown to possess self-similar structure [56] and their function as Josephson junctions possessing higher-order geometric properties in vivo could be seen to support "readout" of information as bioactivity, in diverse settings. This type of "biological" JJ would acquire directional character, typical of standard solid-state versions, only as stresses in the wound environment exert field influences able to establish it, based on healing requirements.

It is noteworthy that clotting factors V and VIII are related to Ceruloplasmin, a multicopper oxidase of the blue type [57], factors V and VIII bind copper [58], and copper can potentiate association of factor VIII heavy and light chains [58]. Factors V and VIII are also blue copper oxidases [59]. A report on the primary structure of ascorbate oxidase concluded that the small blue copper proteins likely evolved from the same ancestral gene as the multicopper oxidases [60]. Moreover, cysteine has been described as "obligatory" in formation of a blue site [61], and this author presents a theory of "rack induced bonding," to explain high reduction potentials of these proteins, necessitating cooperative interactions of multiple influences around the active site, including the ligands themselves [61]. Cysteine residues can be acted on by $\mathrm{H}_{2} \mathrm{O}_{2}$, a product of the amine oxidase reaction, to become "direct" sensors of redox status [62] with obvious advantages in terms of energy cost and speed, over redox sensing mechanisms requiring post-translational modifications. These principles could explain the necessity for proteins, such as Cystatin c, to be called into service in support of local CDAO function for clearance of potent amines in settings of dynamic stress, and consequent changes in the solvent environment.

Heme-oxygenase (HO) is competitively inhibited by calveolin-1 (Cav-1) [63], a protein recently described for a role in hereditary pulmonary arterial hypertension $(\mathrm{HPAH}$, possessing a conserved cysteine near a frameshift mutation associated with two cases of the disease [64]. The redox sensitivity of this residue (cys-156) was demonstrated by Snitrosation resulting from TNF or NO donor application that lead to rapid degradation of the protein [65]. HO is known for a critical role in the turnover of red blood cells, and a capacity to utilize heme as a prosthetic group or a substrate [66]. As pulmonary hypertension (PH) is a condition widelyknown to be caused by abuse of amphetamines, the role of this redox sensitive Cys- 156 becomes clear, if Cav-1 is a 
CDAO of the small blue copper type, and the mutations led to elevations in potent amines. Moreover, specific binding of Cav-1 and HO [63] could amount to formation of a dual $\mathrm{Cu} / \mathrm{Fe}$ dependent oxygen sensing apparatus at wound sites, explaining the competitive inhibition of $\mathrm{HO}$ by Cav-1, based on a finite local oxygen level. Accordingly, an evolved relationship between these two proteins could support their assembly in times of stress to facilitate a dynamic geometric relationship between iron and copper, necessary for gating and orientation of electron flow from oxygen, dependent on the mirage effect, levels of potent amines, redox status, [O2] and other local conditions.

ADAMTS13 is a metalloprotease that mediates cleavage of $\mathrm{vwF}$, with a recognized role in syndromes of thromobotic microangiopathy resulting from aberrant polymerization of vwF, and consequent clumping of platelets at sites of high shear stress in the vasculature [67]. This factor contains two "CUB" domains, a motif named for the presence of sequences sharing homology with complement elements C1r/C1s, a sea urchin protein, Uegf, and BMP-1 [67], a protease of the astacin family. Multiple cysteine residues within each of these two domains are required for stability and secretion of ADAMTS13 [68]. It is noteworthy that the astacin family was defined based on the $82 \%$ sequence identity in the 198 aa N-terminals of the human brush border acid hydrolase (PPH) and mouse kidney brush border enzyme (meprin A), with close identity to BMP-1 and astacin, a crayfish digestive protease, with 3 cysteines, among 37 strictly conserved residues, in a frame aligned on the entire 200-aa sequence of astacin [69].

Direct injection of BMP1 and whole CUB domains can dorsalize the ventral half of Xenopus embryos [70], suggesting the importance of these domains in early neural cell fate, as well as orientation of cells in the organism. An astacin family protein from crayfish has shown enhanced activity with Co substitution, and diminished activity with $\mathrm{Cu}$ substitution relative to $\mathrm{Zn}$ [71]. Another astacin family member, Blastula protease 10 (BP10) possesses structural domains similar to BMP-1 and showed a $960 \%$ enhancement in hydrolysis rate of N-benzoyl-arginine-p-nitroanilide when derivatized to $\mathrm{Cu}$, rather than $\mathrm{Zn}$ [72]. These authors note the similarity of the active site and substrate specificity of BP10 to that of Serralysin, a metalloprotease involved with virulence mechanisms of Pseudomonas aeruginosa and Serratia marcescens that have been recently studied [73]. This evidence suggests that regulatory mechanisms dependent on the availability of copper and zinc are a very serious matter for survival of multicellular organisms.

The role of CUB domains in orientation is further suggested by similarities in the interaction of Neuropilin-1 (NRP-1) with Sema3a and plexin that regulates axonal guidance through cytoskeletal influences [74], and the vwF interaction with FVIII critical to regulation of platelet aggregation [67]. The presence of two CUB domains and two FactorV/FactorVIII-like discoidin domains in NRP-1 suggests it may have a role comparable to ADAMTS13 in the vwF axis. A switch in response to Sema3a can be regulated by ADAM metalloproteases [75]. Similarity to the FVIII/vwF/ADAMTS13 axis suggests how formation of Sema3a/NRP-1/Plexin complexes around neurons represents acquisition of a dynamic sensory capacity to "read-in" (or "sense") levels of potent amines, redox status, and other aspects of the local environment, amounting to multiple oriented field influences. A "read-out" of these signs of stress, through influences on the cytoskeleton, either to stabilize the neural network, or destabilize it, and initiate regulation of axon guidance, directly parallels orientation of a "biologic" JJ described earlier for fibrin polymers.

Correlation of elevated RANTES, but not absence of hemorrhage, with survival in pediatric Ebola virus disease [76] may be explained if flow through clot structures serves a vital function, dependent on binding of copper, amounting to transformation of the entire surface area of the vascular wall into a "quantum computing" device highly specific to addressing viremia. The unique capacity of copper, among other transition metals tested, including iron and zinc, to support higher-order oligomerization of RANTES, with maintenance of function in states of redox stress [77], suggests a role in recovery of released copper from clot sites.

Factors V and VIII have also been noted to have complex metal ion requirements for secretion and functionality [78, 79]. Homology of the binding sites for APC in the FVa and FVIII light chain A domains with regions in ceruloplasmin has been interpreted to suggest involvement with ion binding [80]. FV and FVIII have dual sorting signals [78], though these authors describe only a shift from high to low $[\mathrm{Ca}++]$ for relevance in binding to LMAN1 during transit from ER to ER-Golgi intermediate, leaving open the possibility of an unrecognized signal mediating interaction with MCFD2. Evidence that FV and FVIII are blue copper oxidases related to ceruloplasmin suggests that copper is the second signal. Reduction in the activity of APC in the presence of copper, and reversal of inhibition in the presence of human serum albumin (HSA), or a high-affinity copper-binding analogue of HSA [81] reveals a novel mode of FV and FVIII activity regulation, and the potential utility of APC in sepsis to sequester free copper.

The role of copper may also explain the superiority of heparin over protease inhibitors or chelating anticoagulants, in maintaining stability of FVIII procoagulant activity (VIII:C) [82]. Recovery of activity from CPD plasma with recalcification in the presence of heparin plasma suggested restoration was due to renaturation rather than enzymatic action. These authors conclude by emphasizing the value of maintaining physiologic calcium ion availability for preservation of VIII:C activity [82]. Such physiologic environments would likely also support natural copper ion bioavailability.

It has been suggested that statins [76], and specifically Atorvastatin [83], could have value in treating Ebola. Statin treatment for dyslipidemia has been shown to significantly reduce serum zinc, copper and Ceruloplasmin [84]. Atorvastatin treatment improved arterial stiffness in elderly patients in association with a $20 \%$ reduction in von Willebrand factor, a $26.4 \%$ increase in $\mathrm{Cu} / \mathrm{Zn}$ SOD activity [85], and cognition and depression in patients with $\mathrm{AD}$ or MCI in association with reduced ceruloplasmin [86]. Chelating activity of atorvastatin metabolites has been suggested to account for concentration dependent reduction in LDL oxidation by copper [87]. Induction of tissue factor 
expression in human THP-1 monocytic cells by ceruloplasmin or copper further suggests a central regulatory function [88]. The requirement for 8-hydroxyquinoline, a lipophilic chelator, in revealing the effect [88] suggests the difficulty in dissecting the role of copper in ex vivo settings.

Enhancement of tissue factor expression in monocytes by CD40 ligand [89] reveals the role of copper in outcomes of severe bloodborne infection. The finding of elevated sCD40L in survivors, with fatal outcomes correlating with elevated thrombomodulin, ferritin and D-dimer in Ebola infection [90] and vwF with fatal outcome in Sudan virus infection [91] suggest that monocytes act to integrate evidence of platelet activation (sCD40L) with levels of free copper, controlling tissue factor expression and mobilization of factors that can recover copper for the host, such as RANTES, and FV/VIII. Elevation of vwF, the binding partner of FV/FVIII, would suggest a state of depletion of FV/FVIII, with collapse of ability to synthesize these factors as the virus has gotten decisive access to the host's copper, needed at clot sites. D-dimer elevation reflects clot degradation to gain this access. Elevated ferritin would reflect simultaneous release of free iron, the redox cycling partner of copper. Elevation of thrombomodulin may reflect depletion of APC, it's binding partner.

The ability of zinc, copper and calcium to alter the structure and stability of SAA [92] reveals a role for SAA elevation in pediatric EHF cases [76] in support of copper transport. Accordingly, delivery of copper to monocytes explains induction of tissue factor [ref 36, in 76]. Reduction in endothelial NO synthase production and bioavailability of NO [ref 37, in 76], could occur by regulating copperdependent complex formation between eNOS and caveolin-1 that can inhibit NO synthesis [93] in line with the interaction between HO-1 and caveolin-1, described previously. Caveolin-1 has recently been described as an "essential regulator of eNOS" with disruption underlying endothelial dysfunction [94]. This suggests a role for statins, as they can reduce vascular endothelial expression of caveolin-1, thereby increasing eNOS activity in the setting of cardiovascular disease [95]. The subtle chelating action of statins, and factors such as SAA and copper-dependent proteins, suggests that experimental work will be required to reveal optimal ways of achieving a desired effect on eNOS, or other critical activities beneficial to the host dependent on redistribution of copper ions.

\section{CONCLUSION}

Descriptions of Majorana bound modes [14] suggest they arose in the course of cyclic adiabatic processes applied to lysates of human platelets within a defined space. Two entangled initial states were developed based on the asymmetry created by the dialysis membrane and oriented flow of $\mathrm{HCl}$. An initial "stress" created by placement in the larger dilute acid volume could support redistribution of copper ions across the membrane, leading to a transient drop in CDAO activity, and elevation of potent amine levels within the bag. Their redistribution to the larger external volume could account for aspects of the "entangled" states that would eventually stabilize, and support formation of looping currents of such low MW elements between the spaces. Under these conditions the separated states can acquire phase differences, a Pancharatnam-Berry phase, that can be undefined (singular) for some combination of parameters [1]. The Berry phase is described to be independent of the rate the path is traversed [96].

In the system examined, distinct states of matter were defined to exist through rigorous measurement, though their nature was likely initially dependent on more than 2 oriented field influences. These states can be seen as dependent not only on the path taken to create them, but also the rate at which the path was traversed, such as how long they were in contact across the membrane before their fusion, or the interval between lysis and placement in dialysis. Thus, the model provides a way of conceptualizing a rate-dependent phase resulting from an unlimited number of influences, typical of stresses on organisms. The name proposed for this is the Ramis-Ackroyd-Murray-Hudson phase, or Ramis phase for short. Mathematically, this obviates a dynamical phase factor, resulting in an equation of state defined by the Ramis phase in a single exponential term. In this study the phase shift was defined by external dialysis volume, and this parameter alone fully determined the final state, and separation between adjoining stationary states. The sense of being "in the moment," often during intense concentration required for practice of some highly developed and cherished art, where a feeling of timelessness takes over, seamlessly integrating past, present and future in the artist, essential for the manifestation, could be seen to underlie creation and function of these states in humans.

The Ramis phase contained information that became stored in response to stress, retrievable upon fusion of the separated, bound modes, amounting to stable bioactivity, highly specific to countering the stress that created it. Other bioactivities of interest for regenerative or diagnostic purposes could result from this type of system applied to any lysed tissue possessing valuable biosynthetic properties. It should be informative to examine the effect of removing the bag fully, or to varying degrees, from the larger volume, transferring the material to a separate container, and then reintegrating at later times into the larger (low MW portion), as this would be predicted to destroy information nascent between the two, linked modes, maintained by cycling connections across the membrane.

The key to exploiting distinct stresses will be having a relevant assay to establish the appropriate field influences that force the necessary phase shift, and subsequent reduction into two final control parameters that alone can create the shift. In this example these parameters were volume (a geometric parameter) and diffusion (a timedependent parameter), implying that the Pancharatnam-Berry phase drove evolution into the final purely geometric state. It seems intuitive that, generally, one parameter will be dynamic and the other geometric, a signature of the two original stresses in the ecosystem: cyclical sunlight exposure, and gravity. The process of reduction can be seen as finding a path of least action in a process space.

The separation and later integration of egg yolk and white portions in the creation of diverse foods, such as chocolate mousse, can be seen to depend similarly on a path of stresses, and time taken at each step, such as those 
involved with incorporation of ingredients like chocolate, butter, sugar, orange liqueur, and coffee, into the yolk phase, and subsequent integration of the white phase processed by application of a shearing field for the right amount of time to generate proper foam structure. Incorporation of excessively high percentages of cacao $(>62 \%)$ has been observed to consistently disrupt creation of a desirable, smooth final state (author's unpublished observation), suggesting a distinct symmetry breaking effect. This point of breaking may happen at higher percentages of cacao, with application of processes known to more experienced practitioners. The fact that interactions between these same materials in a geometrically confined space results in the birth of chicks under appropriate conditions, should not be missed for relevance to the ideas developed.

The "create-braid-measure" paradigm of Hasan and Kane [97] can be seen to have occurred in the system studied wherein creation of "entangled" separation supports braiding of vortices set up across the membrane with consequent "quantum computation"-like events. Measurement of the states occurred when brought back together into a stable superposition, locking the information into a new bound state, quantified in the assay system. This information determined viability and phenotypic properties of primary human cells relevant to regenerative responses. The physical and mathematical models presented and discussed in this paper suggest that topologically ordered states forming at non-zero temperature can underlie control of developmental responses in living systems. They may also provide the missing elements in the quest for exact solutions in General Relativity, if the concept of "energy conditions" [98] is broadened to "emergent property conditions" with information flow seen as the regulator of matter-energy transformation necessary for their fulfillment.

Because the states described have been quantified, and assuming they fit a form like the time-independent Schrödinger equation, solutions to the time-dependent form for additional states of the system should be straightforward, and easily generalized to other living systems. Since tensor representations of the Berry curvature can be developed [96], better weighting algorithms for MRI should be possible, if incident and readout magnetic fields are seen to support creation of Ramis phases that contain information retrievable as visual images upon their fusion. These ideas are also expected to be useful in development of methods for channeling sunlight and substrates into plant mashes, plant whole tissue mounts, or microbial culture systems requiring determination of eigenmodes of orientation with respect to gravity, sunlight, and substrate dosing able to lock these systems into states of desired biosynthetic behavior, such as may be necessary for confinement and isolation of metallic hydrogen phases.

\section{CONFLICT OF INTEREST}

The author confirms that this article content has no conflict of interest.

\section{ACKNOWLEDGEMENTS}

The author acknowledges the kind assistance of James West, $\mathrm{PhD}$ for providing access to his lab at Vanderbilt
University so the extracts could be made, putting together Fig. (1), and the material resources for these experiments, as well as the kind assistance of Tom Blackwell, for handling the cell cultures, the MTT assay and representative photo collection that was essential for proper blinding prior to analysis of the data. Dr. West and Steve Simske PhD are both acknowledged for assistance in obtaining and sharing access to referenced papers the author could not have otherwise obtained.

\section{REFERENCES}

[1] http://en.wikipedia.org/wiki/Geometric_phase; [accessed online March 26, 2015].

[2] Nurden AT. Platelets, Inflammation and tissue regeneration. Thromb Haemost 2011; 105 (Suppl 1): S13-33.

[3] Roedersheimer MT, inventor; The Regents of the University of Colorado, a body corporate, assignee. Methods for extracting platelets and compositions obtained therefrom. United States patent US 20100222253. 13 April.

[4] Roedersheimer M, Nijmeh H, Burns N, Sidiakova AA, Stenmark KR, Gerasimovskaya EV. Complementary effects of extracellular nucleotides and platelet-derived extracts on angiogenesis of vasa vasorum endothelial cells in vitro and subcutaneous Matrigel plugs in vivo. Vasc Cell 2011; 3(1): 4. doi: 10.1186/2045824X-3-4.

[5] Roedersheimer MT, Bateman TA, Simske SJ. Effect of gravity and diffusion interface proximity on the morphology of collagen gels. $\mathbf{J}$ Biomed Mater Res 1997; 37: 276-81.

[6] Sipe JB, Zhang J, Waits C, Skikne B, Garimella R, Anderson HC. Localization of bone morphogenetic proteins (BMPs)-2, -4 , and -6 within megakaryocytes and platelets. Bone 2004; 35(6): 1316-22.

[7] Burt DW, Law AS. Evolution of the transforming growth factorbeta superfamily. Prog Growth Factor Res 1994; 5(1): 99-118.

[8] Kalen A, Wahlstrom O, Linder CH, Magnusson P. The content of bone morphogenetic proteins in platelets varies greatly between different platelet donors. Biochem Biophys Res Commun 2008; 375(2): 261-4.

[9] Robertson IB, Rifkin DB. Unchaining the beast; insights from structural and evolutionary studies on TGFbeta secretion, sequestration, and activation. Cytokine Growth Factor Rev 2013; 24(4): 355-72.

[10] Bragdon B, Moseychuk O, Saldanha S, King D, Julian J, Nohe A. Bone Morphogenetic Proteins: a critical review. Cell Signal 2011; 23: 609-20.

[11] Sampath TK, Rashka KE, Doctor JS, Tucker RF, Hoffmann FM. Drosophila transforming growth factor beta superfamily proteins induce endochondral bone formation in mammals. Proc Natl Acad Sci USA 1993; 90(13): 6004-8.

[12] [accessed online March 28, 2015] http://en.wikipedia.org/ wiki/High-temperature_superconductivity\#Examples

[13] Desiderio MA, Zini I, Davalli P, et al. Polyamines, ornithine decarboxylase, and diamine oxidase in the substantia nigra and striatum of the male rat after hemitransection. J Neurochem 1988; 51(1): 25-31.

[14] Alicea J. New directions in the pursuit of Majorana fermions in solid state systems. Rep Prog Phys 2012 (75): 076501. doi: 10.1088/0034-4885/75/7/076501.

[15] [accessed online March 26, 2015] http://www.colorado.edu/ physics/2000/bec/lascool4.html

[16] Ashcroft NW. Metallic Hydrogen: A High-Temperature Superconductor? Phys Rev Lett 1968; 21: 1748.

[17] Ohno K, Ito M, Ichihara M, Ito M. Molecular hydrogen as an emerging therapeutic medical gas for neurodegenerative and other diseases. Oxid Med Cell Longev 2012; 2012: 353152. doi: $10.1155 / 2012 / 353152$.

[18] [accessed online March 28, 2015] http://en.wikipedia.org/wiki/ Heavy_water\#Effect_on_biological_systems

[19] Robinson MM, Turner SM, Hellerstein MK, Hamilton KL, Miller BF. Long-term synthesis rates of skeletal muscle DNA and protein are higher during aerobic training in older humans than in sedentary young subjects but are not altered by protein supplementation. FASEB J 2011; 25(9): 3240-9. 
[20] Crommie MF, Lutz CP, Eigler DM. Confinement of electrons to quantum corrals on a metal surface. Science 1993; 262(5131): 21820 .

[21] Tsukihara T, Aoyama H, Yamashita E, et al. Structures of metal sites of oxidized bovine heart cytochrome $\mathrm{C}$ oxidase at $2.8 \mathrm{~A}$. Science 1995; 269(5227): 1069-74.

[22] Duce JA, Ayton S, Miller AA, et al. Amine oxidase activity of $\beta$ amyloid precursor protein modulates systemic and local catecholamine levels. Mol Psychiatry 2013; 18(2): 245-54.

[23] Bund T, Boggs JM, Harauz G, Hellmann N, Hinderberger D. Copper uptake induces self-assembly of $18.5 \mathrm{kDa}$ myelin basic protein (MBP). Biophys J 2010; 99(9): 3020-8.

[24] Tavassoly O, Nokhrin S, Dmitriev OY, Lee JS. Cu(II) and dopamine bind to alpha-synuclein and cause large conformational changes. FEBS J 2014; 281(12): 2738-53.

[25] Liu L, Jiang D, McDonald A, Hao Y, Millhauser GL, Zhou F. Copper redox cycling in the prion protein depends critically on binding mode. J Am Chem Soc 2011; 133(31): 12229-37.

[26] Fiete GA, Heller EJ. Theory of quantum corrals and quantum mirages. Rev Mod Phys 2003; 75: 933; [accessed online March 28, 2015] http://arxiv.org/pdf/cond-mat/0211607.pdf

[27] Goldwasser E, Jacobson LO, Fried W, Plzak LF. Studies on erythropoiesis. V. The effect of cobalt on the production of erythropoietin. Blood 1958; 13(1): 55-60.

[28] Chandel NS, Maltepe E, Goldwasser E, Mathieu CE, Simon MC, Schumacker PT. Mitochondrial reactive oxygen species trigger hypoxia-induced transcription. Proc Natl Acad Sci USA 1998; 95(20): 11715-20.

[29] Kobayashi M, Shimizu S. Cobalt proteins. Eur J Biochem 1999; 261(1): 1-9.

[30] Lynn JW, Huang Q, Brown CM, et al. Structure and Dynamics of Superconducting $\mathrm{NaxCoO}_{2}$ Hydrate and it's unhydrated analog. Phys Rev B 2003; 68: 214516; [accessed March 28, 2015] http://arxiv.org/pdf/cond-mat/0307263v2.pdf

[31] Teufel JS. PhD dissertation, Experimental investigation of H2/D2 isotope separation by cryo-absorption in metal-organic frameworks. Max-Planck-Institute fur Intelligente Systeme, published April 10, 2013 [accessed March 28, 2015] http:// elib.unistuttgart.de/opus/volltexte/2013/8122/pdf/Dissertation_Julia_Teufe 1_final_4.pdf

[32] Kelton JG, Steeves K. The amount of platelet-bound albumin parallels the amount of IgG on washed platelets from patients with immune thrombocytopenia. Blood 1983; 62(4): 924-7.

[33] Rhee S, Grinnell F. Fibroblast mechanics in 3D collagen matrices. Adv Drug Deliv Rev 2007; 59(13): 1299-305.

[34] Betz P, Nerlich A, Wilske J, Tubel J, Penning R, Eisenmenger W. Immunohistochemical localization of collagen types I and VI in human skin wounds. Int J Legal Med 1993; 106(1): 31-4.

[35] Rinn JL, Wang JK, Liu H, Montgomery K, van de Rijn M, Chang HY. A systems biology approach to anatomic diversity of skin. J Invest Dermatol 2008; 128(4): 776-82.

[36] Berridge MV, Herst PM, Tan AS. Tetrazolium dyes as tools in cell biology: new insights into their cellular reduction. Biotechnol Annu Rev 2005; 11: 127-52.

[37] [accessed March 28, 2015] http://www.nobelprize.org/nobel_ prizes/chemistry/laureates/1907/buchner-bio.html

[38] Geshkenbein BV, Larkin AI. Vortices with half magnetic flux quanta in "heavy-fermion" superconductors. Phys Rev B 1987; 36(1): 235-8.

[39] Uji S, Terashima T, Nishimura M, et al. Vortex dynamics and the Fulde-Ferrell-Larkin-Ovchinnikov State in a Magnetic-FieldInduced Organic Superconductor. Phys Rev Lett 2006; 97: 157001.

[40] Reimers JR, Mckemmish LK, McKenzie RH, Mark AE, Hush NS. Weak, strong, and coherent regimes of Fröhlich condensation and their applications to terahertz medicine and quantum consciousness 2009; 106(11): 4219-24.

[41] Gritsev, V Polkovnikov A. Dynamical quantum Hall effect in the parameter space. Proc Natl Acad Sci USA 2012; 109(17): 6457-62.

[42] [accessed March 28, 2015] http://en.wikipedia.org/wiki/Susceptibility

[43] Avnir D, Kagan ML. The evolution of chemical patterns in reactive liquids driven by hydrodynamic instabilities. CHAOS 1995; 5(3): 589-601.

[44] Kagan ML, Kepler TB, Epstein IR. Geometric phase shifts in chemical oscillators. Nature 1991; 349(6309): 506-8.
[45] Garneata L, Slusanschi O, Preoteasa E, Corbu-Stancu A, Mircescu G. Periodontal status, inflammation, and malnutrition in hemodialysis patients - is there a link? J Ren Nutr 2015; 25(1): 67-74.

[46] Marsenic O, Wierenga A, Wilson DR, et al. Cystatin C in children on chronic hemodialysis. Pediatr Nephrol 2013; 28(4): 647-53.

[47] Oh MY, Lee H, Kim JS, et al. Cystatin C, a novel indicator of renal function, reflects severity of cerebral microbleeds. BMC Neurol 2014; 14: 127. doi: 10.1186/1471-2377-14-127.

[48] Watanabe S, Hayakawa T, Wakasugi K, Yamanaka K. Cystatin C protects neuronal cells against mutant copper-zinc superoxide dismutase-mediated toxicity. Cell Death Dis 2014; 5, e1497; doi:10.1038/cddis.2014.459

[49] Kaur G. Levy E. Cystatin C in Alzheimer's Disease. Front Mol Neurosci. 2012; 5:79. doi: 10.3389/fnmol.2012.00079.

[50] Januszewski AS, Mason N, Karschimkus CS, et al. Plasma semicarbazide-sensitive amine oxidase activity in type 1 diabetes is related to vascular and renal function but not to glycaemia. Diab Vasc Dis Res 2014; 11(4): 262-9.

[51] Noonan T, Lukas S, Peet GW, et al. The oxidase activity of vascular adhesion protein-1 (VAP-1) is essential for function. Am J Clin Exp Immunol 2013; 2(2): 172-185.

[52] Somfal GM, Knippel B, Ruzicska E, et al. Soluble semicarbazidesensitive amine oxidase (SSAO) activity is related to oxidative stress and subchronic inflammation in streptazotocin-induced diabetic rats. Neurochem Int 2006; 48(8): 746-52.

[53] Zerovnik E, Skerget K, Tusek-Znidaric M, Loeschner C, Brazier MW, Brown DR. High affinity copper binding by stefin B (cystatin B) and its role in the inhibition of amyloid fibrillation. FEBS $J$ 2006; 273(18): 4250-63

[54] Kaur G, Mohan P, Pawlik M, et al. Cystatin C rescues degenerating neurons in a cystatin B-knockout mouse model of progressive myoclonus. Am J Pathol 2010; 177(5): 2256-67.

[55] Davalos D, Ryu JK, Merlini M, et al. Fibrinogen-induced perivascular microglial clustering is required for the development of axonal damage in neuroinflammation. Nat Commun 2012; 3: 1227. doi: 10.1038/ncomms2230.

[56] Kubota K, Kogure H, Masuda Y, et al. Gelation dynamics and gel structure of fibrinogen. Colloids Surf B Biointerfaces 2004; 38(34): 103-9.

[57] Pisu P, Bellovino D, Gaetani S. Copper regulated synthesis, secretion and degradation of ceruloplasmin in a mouse immortalized hepatocytic cell line. Cell Mol Biol (Noisy-le-grand) 2005; Suppl 51: OL859-67.

[58] Milne DB Nielsen FH. Effects of a diet low in copper on copperstatus indicators in postmenopausal women. Am J Clin Nutr 1996; 63: 358-64.

[59] Ryden LG, Hunt LT. Evolution of protein complexity: The blue copper-containing oxidases and related proteins. J Mol Evol 1993; 36(1): 41-66.

[60] Ohkawa J, Okada N, Shinmyo A, Takano M. Primary structure of cucumber (Cucumis sativus) ascorbate oxidase deduced from cDNA sequence: Homology with blue copper proteins and tissuespecific expression. Proc Natl Acad Sci USA 1989; 86(4): 1239-43.

[61] Malmstrom BG. Rack-induced bonding in blue-copper proteins. Eur J Biochem 1994; 223(3): 711-8

[62] Benoit R, Auer M. A direct way of redox sensing. RNA Biol 2011; 8(1): 18-23.

[63] Taira J, Sugishima M, Kida Y, Oda E, Noguchi M, Higashimoto Y. Caveolin-1 is a competitive inhibitor of heme oxygenase-1 (HO-1) with heme: identification of a minimum sequence in caveolin- 1 for binding to HO-1. Biochemistry 2011; 50(32): 6824-31.

[64] Austin ED, Ma L, LeDuc C, et al. Whole exome sequencing to identify a novel gene (caveolin-1) associated with human pulmonary arterial hypertension. Circ Cardiovasc Genet 2012; 5(3): 336-43.

[65] Bakhshi FR, Mao M, Shajahan AN, et al. Nitrosation-dependent caveolin I phosphorylation, ubiquitination, and degradation and its association with idiopathic pulmonary arterial hypertension. Pulm Circ 2013; 3(4): 816-30.

[66] Fraser ST, Midwinter RG, Berger BS, Stocker R. Heme Oxygenase-1: a critical link between iron metabolism, erythropoiesis, and development. Adv Hematol 2011; 2011; 473709. doi: 10.1155/2011/473709.

[67] Lancellotti S, Basso M, De Cristofaro R. Proteolytic processing of von Willebrand factor by adamts 13 and leukocyte proteases. Mediterr J Hematol Infect Dis 2013; 5(1): e2013058. 
[68] Zhou Z, Yeh HC, Jing H, et al. Cysteine residues in CUB-1 domain are critical for ADAMTS13 secretion and stability. Thromb Haemost 2011; 105(1): 21-30.

[69] Dumermuth E, Sterchi EE, Jiang WP, et al. The Astacin family of metalloendopeptidases. J Biol Chem 1991; 266(32): 21381-5.

[70] Lee HX, Mendes FA, Plouhinec JL, De Robertis EM. Enzymatic regulation of pattern: BMP4 binds CUB domains of Tolloid and inhibits proteinase activity. Genes Dev 2009; 23(21): 2551-62.

[71] Gomis-Ruth FX, Grams F, Yiallouros I, et al. Crystal structure, spectroscopic features, and catalytic properties of Cobalt(II), Nickel(II), and Mercury(II) derivatives of the zinc endopeptidase Astacin. J Biol Chem 1994; 269(25): 17111-7.

[72] da Silva GF, Reuille RL, Ming LJ, Livingston BT. Overexpression and mechanistic characterization of blatula protease 10, a metalloprotease involved in Sea Urchin embryogenesis and development. J Biol Chem 2006; 281(16): 10737-44.

[73] Butterworth MB, Zhang L, Liu X, Shanks RM, Thibodeau PH. Modulation of the epithelial sodium channel $(\mathrm{ENaC})$ by bacterial metalloproteases and protease inhibitors. PLoS One 2014; 9(6): e100313.

[74] Nakamura F, Kalb RG, Strittmatter SM. Molecular basis of semaphorin-mediated axon guidance. J Neurobiol 2000; 44(2): 219-29.

[75] Romi E, Gokhman I, Wong E, et al. ADAM metalloproteases promote a developmental switch in responsiveness to axonal repellant Sema3a. Nat Commun 2014; 5: 405. doi: 10.1038/ncomms 5058.

[76] McElroy AK, Erickson BR, Flietstra TD, et al. Biomarker correlates of survival in pediatric patients with ebola virus disease. Emerg Infect Dis 2014; 20(10): 1683-90.

[77] MacGregor HJ, Kato Y, Marshall LJ, Nevell TG, Shute JK. A copper-hydrogen peroxide redox system induces dityrosine crosslinks and chemokine oligomerisation. Cytokine 2011; 56(3): 66975 .

[78] Zheng C, Zhang B. Combined deficiency of coagulation factors V and VIII: an update. Semin Thromb Hemost 2013; 39(6): 613-20.

[79] Pitman DD, Tomkinson KN, Kaufman RJ. Post-translational requirements for functional factor $\mathrm{V}$ and factor VIII secretion in mammalian cells. J Biol Chem 1994; 269(25): 17329-37.

[80] Walker FJ, Scandella D, Fay PJ. Identification of the binding site for activated protein $\mathrm{C}$ on the light chain of factors $\mathrm{V}$ and VIII. $\mathrm{J}$ Biol Chem 1990; 265(3): 1484-9.

[81] Bar-Or D, Rael LT, Winkler JV, Yukl RL, Thomas GW, Shimonkevitz RP. Copper inhibits activated protein C: protective effect of human albumin and an analogue of its high affinity copper-binding site, d-DAHK. Biochem Biophys Res Commun 2002; 290(5): 1388-92.

[82] Rock GA, Cruickshank WH, Tackberry ES, Ganz PR, Palmer DS. Stability of VIII:C in plasma: the dependence on protease activity and calcium. Thromb Res 1983; 29(5): 521-35.
[83] Fedson DS. A practical treatment for patients with Ebola virus disease. J Infect Dis 2015; 211(4): 661-2.

[84] Ghayour-Mobarhan M, Lamb DJ, Taylor A, et al. Effect of statin therapy on serum trace element status in dyslipidaemic subjects. J Trace Elem Med Biol 2005; 19(1): 61-7.

[85] Wang J, Xu J, Zhou C, et al. Improvement of arterial stiffness by reducing oxidative stress damage in elderly hypertensive patients after 6 months of atorvastatin therapy. J Clin Hypertens (Greenwich) 2012; 14(4): 245-9.

[86] Sparks DL, Petanceska S, Sabbagh M, et al. Cholesterol, copper and Abeta in controls, MCI, AD and the AD cholesterol lowering treatment trial (ADCLT). Curr Alzheimer Res 2005; 2(5): 527-39.

[87] Aviram M, Rosenblat M, Bisgaier CL, Newton RS. Atorvastatin and gemfibrozil metabolites, but not the parent drugs, are potent antioxidants against lipoprotein oxidation. Atherosclerosis 1998; 138(2): 271-80.

[88] Crutchley DJ. Que BG. Copper-induced tissue factor expression in human monocytic THP-1 cells and its inhibition by antioxidants. Circulation 1995; 92(2): 238-43

[89] Sanguigni V, Ferro D, Pignatelli P, et al. CD40 ligand enhances monocyte tissue factor expression and thrombin generation via oxidative stress in patients with hypercholesterolemia. J Am Coll Cardiol 2005; 45(1): 35-42

[90] McElroy AK, Erickson BR, Flietstra TD, et al. Ebola hemorrhagic Fever: novel biomarker correlates of clinical outcome. J Infect Dis 2014; 210(4): 558-66.

[91] McElroy AK, Erickson BR, Flietstra TD, et al. Von Willebrand factor is elevated in individuals infected with Sudan virus and is associated with adverse clinical outcomes. Viral Immunol 2015 28(1): 71-3.

[92] Wang L, Colon W. Effect of zinc, copper, and calcium on the structure and stability of serum amyloid A. Biochemistry 2007; 46(18): 5562-9.

[93] Ghosh S, Gachhui R, Crooks C, Wu C, Lisanti MP, Stuehr DJ. Interaction between caveolin-1 and the reductase domain of endothelial nitric oxide synthase. Consequences for catalysis. J Biol Chem 1998; 273(35): 22267-71

[94] Williams JJ, Palmer TM. Cavin-1: caveolae-dependent signaling and cardiovascular disease. Biochem Soc Trans 2014; 42(2): 284-8.

[95] Balakumar P, Kathuria S, Taneja G, Kalra S, Mahadevan N. Is targeting eNOS a key mechanistic insight of cardiovascular defensive potentials of statins? J Mol Call Cardiol 2012; 52(1): 8392.

[96] [accessed March 28, 2015] http://en.wikipedia.org/wiki/Berry_ connection_and_curvature

[97] Hasan, MZ Kane, CL. Topological insulators. Rev Mod Phys 2010; 82: 3045 .

[98] [accessed March 28, 2015] http://en.wikipedia.org/wiki/Exact_ solutions_in_general_relativity\#Difficulties_with_the_definition http://www.superconductors.org/28c_rtsc.htm

(C) Mark Roedersheimer; Licensee Bentham Open

This is an open access article licensed under the terms of the Creative Commons Attribution Non-Commercial License (http://creativecommons.org/licenses/ by-nc/3.0/) 